\title{
Animal Communication Networks
}

Peter McGregor (ed). Cambridge University Press, 2005. 657 Pp.

ISBN 0-521-82361-7 (hardcover)

Seven years ago, in a small tavern in eastern Ontario, a colleague asked me an interesting question about communication networks. At that time, Peter McGregor and his collaborators had formally articulated the idea of communication networks, noting that many animal signals transmit over long distances and that communication is better understood as a process occurring among multiple signalers and receivers (McGregor \& Dablesteen 1996). Several important experiments had been published around the time of our conversation, demonstrating that animals extract information from signaling interactions between others (i.e. eavesdropping; McGregor \& Peake 2000). However, my colleague was concerned that the communication network literature involved only these eavesdropping experiments. He asked, "Is there anything more to the communication network idea beyond eavesdropping?” With the publication of Peter McGregor's edited volume Animal Communication Networks in 2005, we have a definite answer to this question: The communication network model facilitates an improved understanding of a multitude of animal behaviors, including eavesdropping but also a broad spectrum of other receiver and signaler activities.

The book consists of twenty-six chapters, loosely organized into four sections. It begins in familiar territory, with the most thorough reviews to date of eavesdropping behavior (chapters 2, 3) and audience effects (changes in signaling behavior caused by the presence of an audience; chapters 4,5 ). These chapters are especially satisfying because each one is firmly supported by numerous compelling behavioral experiments. I expect these chapters will satisfy even the most skeptical behavioral ecologist that communication networks must be invoked to explain some behaviors that do not fit within a dyadic communication model.

The first section of the book also includes a highly provocative chapter by John Bower on victory displays (post-contest displays which are given by the contest winner but not the contest loser). As with many ideas presented in this book, there are no experimental tests of the idea that victory displays are network-based phenomena, but the chapter provides fertile ground for future research questions. This chapter, in particular, should be required reading for any young graduate students in search of unexplored questions in behavioral ecology. Almost every chapter in the book provides numerous compelling and unanswered research questions which are sure to stimulate future network research. Furthermore, throughout the book there has been a clear effort to depict the primary experimental protocols, often through the use of figures, and this is likely to inspire both lab-based and field-based behavioural ecologists.

The second section of the book deals with communication networks in particular contexts, and the third section of the book deals with communication networks in different taxa. Although birds and fish receive substantial attention throughout the book, intriguing chapters also explore communication networks in insects, fiddler crabs, frogs, rodents, large terrestrial mammals, marine mammals, and even humans. The network model offers insights across many organisms, testifying that the network model is a valuable one for comprehending complex patterns of animal communication. These collected chapters from such a diversity of study systems help the reader to draw general conclusions about the influence of group living on animal signaling behavior.

The fourth and final section of Animal Communication Networks attempts to connect the network idea to other biological disciplines, including animal cognition, the evolution of altruism, and primate sociality. These chapters provide interesting food for thought. For example, Irene Pepperberg points out that the cognitive processes associated with eavesdropping are similar to those associated with transitive inference. Redouan Bshary and Arun D'Souza point out that altruistic behavior is easy to understand within a network context where many individuals are assessing each others' behavior.

How is our understanding of animal communication expanded by adopting a network perspective? McGregor's book provides many examples of how network thinking enhances our understanding of behavioral ecology. Here are three exciting examples. (1) Anahita Kazem and Filippo Aurelli apply the communication network model to redirection-ofaggression behavior in primates (chapter 10). They evaluate whether redirection may serve a signaling function within social groups, as opposed to a 
traditional view of redirection as a venting of frustration. By considering primate behavior within a communication network, the authors realize that redirection might play an important role in pre-empting attacks by bystanders who witness the redirected aggression. (2) Marty Leonard and Andy Horn evaluate nestling begging in birds as a communication network, and confront the complexity of a network that consists of organisms signaling within extremely close proximity. Colorfully describing the nest as "part communication network, part scrum toward the parent," Leonard and Horn point out that the competitive interactions between nestlings, siblings, and parents cannot be understood merely as dyadic processes. (3) Ken Otter and Laurene Ratcliffe, as well as Torben Dabelsteen, discuss how choosy females are expected to behave when assessing male mating signals. They establish contrasting patterns for how females should behave if they sample males individually (i.e. approach each male directly, then assess) versus if they sample males through eavesdropping (i.e. approach a position that facilitates comparison between two or more individuals, then assess). Dabelsteen interprets the data from Bensch and Hasselquist's (1992) study of the movement patterns of female great reed warblers as evidence for the latter type of assessment.

One of the most impressive features of this edited volume is the diversity of topics and organisms which are evaluated from a communication network perspective. There are a few chapters which are only tangentially related to the communication network idea, and there are a few chapters that are highly taxonspecific. Nevertheless, we should commend the editor for including such a broad array of topics and requesting the involvement of so many authors with such diverse backgrounds and diverse study systems.

A recurring theme throughout the book is the importance of the selective pressures of networks in ancestral signaling environments for understanding present signaling behavior. This is most explicitly dealt with among chapters on fish visual signals (chapter 4) and fish olfactory signals (chapter 23), although it also permeates most sections of the book. The cumulative evidence for the influence of networks on signaling and receiving behavior leads the reader to realize that most forms of communication will have been shaped by evolution within a group setting rather than a dyadic setting.

As the first major synthesis on the topic of communication networks, summarizing roughly ten years of research, the book allows for careful articulation of certain key concepts and terms. Tom Peake's comprehensive review of eavesdropping introduces a useful distinction between two types of eavesdropping. He proposes the term interceptive eavesdropping for the behavior of extracting absolute information from an interaction between others (e.g. a snake locating prey by listening to foot-drumming interactions between kangaroo rats) but distinguishes social eavesdropping for the behavior of extracting relative information from a signaling interaction between others (e.g. a female comparing the relative quality of two males based on their relative vocal performance in a song contest).

Given the breadth of topics covered in the book, Animal Communication Networks serves as a useful textbook. In the fall of 2005, I used this book as the core text for a graduate course in behavioral ecology. The diversity of topics covered within this volume provided an excellent starting point for discussions of general topics in behavioral ecology, while allowing the students to evaluate the utility of applying the communication network model to each of these topics.

This is an important book for behavioral ecologists, especially those interested in animal communication. The message that underlies this comprehensive volume is that we can achieve an enhanced comprehension of animal communication by including the broader social context in which communication occurs.

\section{Daniel Mennill \\ Department of Biological Sciences \\ University of Windsor \\ Ontario, Canada}

\section{References}

Bensch S, Hasselquist D. 1992. Evidence for active female choice in a polygynous warbler. Anim Behav 44:301-311

McGregor PK, Dabelsteen T. 1996. Communication networks. In: Ecology and evolution of acoustic communication in birds (Kroodsma DE, Miller EH, eds) Ithaca: Cornell University Press; 409-425

McGregor PK, Peake TM. 2000.Communication networks: social environments for receiving and signalling behaviour. Acta Ethol 2:71-81 